\title{
GCU
}

Glasgow Caledonian

University

University for the Common Good

\section{Robust disturbance rejection for discrete-time systems having magnitude and rate bounded inputs}

Kucukdemiral, Ibrahim B.

Published in:

The Journal of the Franklin Institute

DOI:

10.1016/j.jfranklin.2020.06.001

Publication date:

2020

Document Version

Author accepted manuscript

Link to publication in ResearchOnline

Citation for published version (Harvard):

Kucukdemiral, IB 2020, 'Robust disturbance rejection for discrete-time systems having magnitude and rate bounded inputs', The Journal of the Franklin Institute, vol. 357, no. 12, pp. 8252-8276.

https://doi.org/10.1016/j.jfranklin.2020.06.001

\section{General rights}

Copyright and moral rights for the publications made accessible in the public portal are retained by the authors and/or other copyright owners and it is a condition of accessing publications that users recognise and abide by the legal requirements associated with these rights.

Take down policy

If you believe that this document breaches copyright please view our takedown policy at https://edshare.gcu.ac.uk/id/eprint/5179 for details of how to contact us. 


\title{
Robust Disturbance Rejection for Discrete-Time Systems having Magnitude and Rate Bounded Inputs
}

\author{
İbrahim B. Küçükdemiral ${ }^{1}$ \\ Glasgow Caledonian University, School of Computing, Engineering and Built Environment, \\ Department of Applied Science, G4 OBA Glasgow, Scotland, UK
}

\begin{abstract}
The design of a robust controller for uncertain discrete-time systems subject to bounded disturbances and having magnitude and rate bounded actuators is considered. To tackle the problem, the proposed method utilises nested ellipsoids and employs an incremental (velocity) form of the state-space model of the system. By using modified full block S-procedure, the proposed robust control design approach is applicable to any uncertain system with rational parameter dependence which cannot be managed by the existing approaches without introducing some extra conservatism. Finally, an algorithm that allows the method to be used as a gain-scheduling controller is also noted in the study.

Keywords: Disturbance rejection, attractive ellipsoid, invariant ellipsoids, linear matrix inequalities, robust control, gain-scheduling control, full-block s-procedure.
\end{abstract}

\section{Introduction}

Almost all practical systems in industry are dominated by physical constraints, subject to uncertainties and detrimental disturbances. Unfortunately, in most cases, these physical constraints are ignored during the design of con-

5 trol systems. One of the most commonly encountered constraints in engineering is the one that is relevant to actuators. The magnitude, especially, the rate bounded actuators are very common, even inevitable in control systems and identified as a source of severe performance degradation or instability in many applications including aerospace and transportation systems, particularly those

10 having mechanical actuators. They may cause fatal effects in several situations such as the crash of YF-22 in 1992, which has been caused by pilot-induced oscillations due to rate saturated control surfaces. Details about these incidents can be found in the references [1, 2], 3] and [4. Other examples of practical problems in which the magnitude and rate bounded actuators create a source

\footnotetext{
${ }^{1}$ E-mail:ibrahim.kucukdemiral@gcu.ac.uk
} 
15 of performance degradation and instability can be listed as the control of jet engine compressors [5], general reaction processes having sluggish actuators [6] and stabilisation of ships using active fins [7. Therefore, designing effective controllers for systems having hard limitations on their actuators and subject to uncertainties and disturbances has been a fundamental problem for the con-

20 trol community for several decades [8, [9]. For those systems having only the magnitude bounded actuators, the literature is immense and the problem is generally solved by using anti-windup controllers. While a comprehensive review of anti-windup techniques is not feasible here, interested readers can consult [10], [11] or [12 and the references therein. As opposed to the broad literature on

25 the control of systems having only magnitude bounded actuators, the research on the control of systems having both magnitude and rate bounded actuators is shallow. In literature, the problem is usually addressed in two main groups, namely nonlinear and linear control settings. In the nonlinear framework, for instance, [13] considers a continuous-time predictive control approach to derive the nonlinear constrained control law for magnitude and rate bounded systems. A nonlinear control method based on Linear Parameter Varying (LPV) modelling is addressed in reference [14] whereas a nonlinear attractive ellipsoid method is proposed in [15. Finally, a nonlinear state feedback control law which facilitates faster convergence compared to linear state feedback while guaranteeing

35 domains of attraction of similar size is taken into account in [16]. In this work, the controller is parametrized by solving a convex optimization problem based on Linear Matrix Inequalities (LMIs).

On the other hand, the linear control solution to the aforementioned problem in literature can be classified into several categories. One group treats

40 the problem in model-predictive control (MPC) framework. Although MPC is a well-established approach for dealing with multi-input, multi-output processes having multiple input/output constraints, its highly complex structure, associated stability problems and requirement for huge real-time computational resources, make it unsuitable to use in applications requiring fast, cheap and 45 time-critical controllers, especially the ones having uncertainties [17]. For this group of studies, a very recent paper [18] worths mentioning as it proposes an MPC topology with comprises a novel discrete-time reduced-order generalized proportional-integral observer (GPIO) to estimate the virtual system states and the lumped disturbances.

${ }_{50} \quad$ The second group involves approaches which can be considered as derivatives of anti-windup strategies that are originally developed for magnitude bounded systems. In anti-windup techniques, input constraints are not directly considered during the controller design, but mainly used on attenuating the unfavourable effects afterwards by introducing compensation components in the

55 closed loop. For instance, in reference [19], a technique which utilises saturating integral blocks and anti-windup loops is proposed to overcome the magnitude and rate saturation problem. Then, $\mathcal{L}_{2}$ gain between the disturbance input and the plant output is considered as a performance measure to be minimized by control. However, this paper does not consider any uncertainty in system ${ }_{60}$ parameters and the proposed technique is developed for a continuous-time pro- 
cess. In reference [20], authors propose a strictly proper controller that utilises derivative of the output signal together within anti-windup context. The note makes use of generalised sector conditions and LMI based tools to provide a solution to the problem. Again, the method is only applicable to continuous-time 65 processes and does not consider the robustness issue. Reference 21 introduces a new, less conservative anti-wind up based controller scheme for magnitude and rate bounded continuous-time systems subject to peak-bounded disturbances. To reduce conservatism and to obtain better performance, a multi-stage antiwind up mechanism is introduced for different levels of saturation. However, 70 the robustness of the method is not studied.

Another group of studies tackles the problem by representing the saturation nonlinearity with a convex hull. This technique handles the magnitude and rate bounds as nested saturation blocks. For instance, one can refer to references [22, 23, 24] and 25] for details and modifications of the approach. Among

75 these literature, 23] and 25] are the only ones that deal with the problem in the discrete-time domain. However, these works do not consider the disturbance rejection problem. The last group of studies tackles the problem by representing the actuator dynamics with a first or higher order model and then deriving controller based on cost functions such as energy-to-energy, energy-to-peak or 80 peak-to-peak gains of the system [26, 27], 28]. However, all of these results are obtained for continuous-time processes and none of them considers robustness issue.

As described above, there is a rich body of work in literature addressing only the problem of magnitude constraints and very limited number of sources 85 dealing with magnitude and rate bounded systems. Within this limited number of works that deal with the latter problem, most of the results are obtained in continuous-time domain and based on anti-wind up technique or its derivatives which rarely consider the robustness issue. Therefore, this study aims to develop a novel, practically applicable robust discrete-time controller for systems having 90 rate and magnitude bounded actuators and subject to bounded disturbances. The contribution of this work to the literature is versatile. First, different from the literature, the proposed method neither relies on anti-windup structure nor on nested saturation blocks but utilizes newly developed nested attractive and invariant ellipsoids 29] which allow us to use efficient LMI tools. The ellipsoid 95 method is based on the well-known Lyapunov method, so it is easy to use other performance measures and methods developed using this instrument. On the other hand, the results of this note are obtained using the so-called velocity form [30] of the augmented system which makes it easy to tackle zero offset reference tracking problem. Moreover, we address a robust controller synthe100 sis method based on the modified full block S-procedure to obtain a controller with minimal conservatism [31. Thanks to the linear fractional representation (LFR) of the uncertain plant and Pólya-type relaxation, the proposed robust control technique is applicable to any uncertain system with rational parameter dependence which cannot be solved by the existing approaches without intro105 ducing extra conservatism. Furthermore, though not considered in this study, the LFR makes the problem to be readily converted to a linear parameter vary- 
ing (LPV) control when the uncertainties are replaced with measured/calculated time-varying parameters of the system. Additionally, usage of extended LMI conditions in the design makes the controller independent of Lyapunov matrices which allows the approach to be used effectively in multi-objective control design with minimal additional conservatism. Finally, the proposed technique of this note adapted to a gain-scheduling control method to obtain a better and aggressive performance from the controller.

The rest of the paper is organised as follows: Section 2 states the problem. In section 3. we present two different design methods for a nominal system subject to bounded disturbances where the first one is proposed for systems having zero initial conditions and the second one is introduced for systems having non-zero initial states. A discussion on how to find optimal attractive and invariant ellipsoids using convex optimisation techniques is presented in Section 4 . Section 5 and its subsections are devoted to the synthesis of robust controllers for systems having perturbed parameters. Based on the proposed methods, Section 6 presents a gain-scheduled controller design approach for systems having hard (rate and/or magnitude type) input constraints. In Section 7, we present the application results of the proposed methods on an illustrative example to draw 125 some final conclusions in Section 8 .

Notation. Most of the notations used are standard. $\mathbb{N}$ and $\mathbb{R}$ stand for the set of natural and real numbers, respectively. $\mathbb{R}^{+}$denotes the set of strictly positive real scalars. $\mathbb{R}^{n}$ shows $n$-dimensional real vectors whereas $\mathbb{R}^{n \times m}$ stands for $n \times m$ real matrices. Notation $u_{k}$ is used to represent a signal $u$ at sampling 130 instant $k$. $I$ represents identity matrix with an appropriate dimension whereas $G^{T}$ represents the transpose of $G$. $\operatorname{He}\{P\}=P+P^{T}$. The symbol $*$ in square matrices denotes symmetric blocks in a symmetric matrix. $\wedge$ symbolises the logical and operator. The notation $u_{i, k}$ is used to show the $i$-th entry of the signal $u$ at the sampling time $k$, whereas $\bar{u}_{i}$ and $\bar{v}_{i}$ represent the scalars that correspond to known physical magnitude and rate bounds for the $i$-th channel of the control signal $u$, respectively. For a vector $u,\|u\|_{2}$ represents the 2-norm of $u$. $\operatorname{diag}\{\cdot\}$ represents block diagonal matrices. Trace $(\cdot)$ shows the trace of a matrix. Finally, the notation $A \succeq(\preceq) 0$ means $A$ is a symmetric positive (negative) semi-definite matrix.

\section{Problem definition}

Consider a discrete-time uncertain plant governed by the state-space equation as follows:

$$
x_{k+1}=A\left(\Delta_{k}\right) x_{k}+B\left(\Delta_{k}\right) u_{k}+H\left(\Delta_{k}\right) w_{k} .
$$

Here, $k \geq 0 \in \mathbb{N}$ is the sampling instant, $x_{k} \in \mathbb{R}^{n}$ is the fully measurable state vector and $w_{k} \in \mathbb{R}^{p}$ is the bounded unknown disturbance signal which 145 satisfies $w_{k}^{T} w_{k} \leq w_{\infty}^{2}$ for all $k \geq 0$ with the known peak value $w_{\infty} \in \mathbb{R}^{+}$. $A\left(\Delta_{k}\right), B\left(\Delta_{k}\right)$ and $H\left(\Delta_{k}\right)$ are uncertain, possibly time-varying, state-space 
matrices in appropriate dimensions where $\Delta_{k} \in \boldsymbol{\Delta}$ represents the time-varying unknown parameter matrix at the time instant $k \geq 0$ and $\boldsymbol{\Delta}$ is a known compact uncertainty set. We assume that $u_{k} \in \mathbb{R}^{m}$ is the control vector whose channels $i=1, \ldots, m$ satisfy the following individual symmetrical constraints:

$$
\left|u_{i, k}\right| \leq \bar{u}_{i}, \quad\left|v_{i, k}\right| \triangleq\left|u_{i, k}-u_{i, k-1}\right| \leq \bar{v}_{i}, u_{i,-1}=0, \quad \forall k \geq 0,
$$

where, $\bar{u}_{i}$ and $\bar{v}_{i}$ are assumed to be symmetric and known magnitude and rate bounds for the $i$-th channel of the control signal, respectively.

The main objective of this note is to find a robust stabilising structured control law for (1) which satisfies the constraints given in (2) and minimizes the effects of disturbance $w_{k}$ on the state vector $x_{k}$ for all $k \geq 0$. The method relies on the full state measurement.

\section{Main results}

In this section, first, we shall consider the nominal case where the system matrices in (1) are all assumed to be known and constant, i.e. $A\left(\Delta_{k}\right)=A$, $B\left(\Delta_{k}\right)=B$ and $H\left(\Delta_{k}\right)=H$. To tackle the problem stated in Section 2, we shall employ invariant and attractive ellipsoids 29. The change in the control signal will be considered as the manipulating variable of the overall system and therefore the actual control signal will be the sum of them. To this end, we introduce the control signal as an artificial state which is also known as the velocity form of the system in the literature [30.

Consider the discrete-time state-space system (1) which is subject to control constraints (2). Note that the control action can always be modelled in statespace as $u_{k}=I u_{k-1}+I v_{k}$ where $u_{-1}=0$ and $v_{k} \triangleq u_{k}-u_{k-1}$ is the rate of control signal at sample $k$. Then, in line with (1), the augmented state-space system can be represented in the velocity form as follows:

$$
\bar{x}_{k+1}=\underbrace{\left[\begin{array}{cc}
A & B \\
0 & I
\end{array}\right]}_{\mathcal{A}} \underbrace{\left[\begin{array}{c}
x_{k} \\
u_{k-1}
\end{array}\right]}_{\bar{x}_{k}}+\underbrace{\left[\begin{array}{c}
B \\
I
\end{array}\right]}_{\mathcal{B}} v_{k}+\underbrace{\left[\begin{array}{c}
H \\
0
\end{array}\right]}_{\mathcal{H}} w_{k} .
$$

The following theorem provides a solution to the problem stated in Section 2 when all initial conditions are zero or very close to zero:

Theorem 1. For a given scalar $r_{1} \in[0,1]$, there exists a stabilizing controller in the form of

$$
u_{k}=u_{k-1}+K\left[\begin{array}{ll}
x_{k}^{T} & u_{k-1}^{T}
\end{array}\right]^{T}, \quad u_{-1}=0, \quad \forall k \geq 0,
$$

for nominal system (1) which is subject to the constraints (2), if there exist $P=P^{T} \in \mathbb{R}^{(n+m) \times(n+m)} \succ 0$, matrices $G \in \mathbb{R}^{(n+m) \times(n+m)}$ and $L \in \mathbb{R}^{m \times(n+m)}$ which satisfy the following linear matrix inequalities:

$$
\left[\begin{array}{ccc}
\left(1-r_{1}\right)\left(G+G^{T}-P\right) & 0 & G^{T} \mathcal{A}^{T}+L^{T} \mathcal{B}^{T} \\
* & r_{1} I & \mathcal{H}^{T} \\
* & * & P
\end{array}\right] \succeq 0
$$




$$
\left[\begin{array}{cc}
G+G^{T}-P & L^{T} \ell_{i} \\
\ell_{i}^{T} L & \left(\frac{\bar{v}_{i}}{w_{\infty}}\right)^{2}
\end{array}\right] \succeq 0, \quad i=1, \ldots, m
$$

and

$$
\left[\begin{array}{cc}
P & P \ell_{i} \\
\ell_{i}^{T} P & \left(\frac{\bar{u}_{i-n}}{w_{\infty}}\right)^{2}
\end{array}\right] \succeq 0, \quad i=n+1, \ldots, n+m,
$$

where $\ell_{i}$ represents a column vector whose elements are equal to zero, except the $i$-th element which is equal to one.

Then, $V_{1}\left(\bar{x}_{k}\right)=\bar{x}^{T} P^{-1} \bar{x} \leq w_{\infty}^{2}$ is a state-invariant attractive ellipsoid for nominal system (1) and the stabilizing controller gain is given by $K=L G^{-1}$.

Proof 1. In the first part of the proof, we shall follow a procedure similar to the one in [32]. Consider a Lyapunov function

$$
V_{1}\left(x_{k}\right) \triangleq \bar{x}_{k}^{T} P^{-1} \bar{x}_{k}, \quad P=P^{T} \succ 0,
$$

along the closed-loop system trajectory

$$
\bar{x}_{k+1}=\underbrace{(\mathcal{A}+\mathcal{B} K)}_{A_{c l}} \bar{x}_{k}+\mathcal{H} w_{k} .
$$

The augmented closed-loop system (9) is stable if

$$
\Delta V_{1}\left(\bar{x}_{k}\right) \triangleq V_{1}\left(\bar{x}_{k+1}\right)-V_{1}\left(\bar{x}_{k}\right) \leq 0,
$$

is satisfied for all $k \geq 0$. Using closed-loop system trajectory (9) in (8), (10) is equivalent to

$$
\left[\begin{array}{c}
\bar{x}_{k} \\
w_{k}
\end{array}\right]^{T}\left[\begin{array}{cc}
A_{c l}^{T} P^{-1} A_{c l}-P^{-1} & A_{c l}^{T} P^{-1} \mathcal{H} \\
* & P^{-1}
\end{array}\right] \underbrace{\left[\begin{array}{c}
\bar{x}_{k} \\
w_{k}
\end{array}\right]}_{z_{k}} \leq 0 .
$$

Adding and subtracting $r_{1} \bar{x}_{k}^{T} P^{-1} \bar{x}_{k}$ and $r_{1} w_{k}^{T} w_{k}$ in the left hand side of the inequality (11), the change in the energy function $V_{1}\left(\bar{x}_{k}\right)$ can be rewritten as

$$
\Delta V_{1}\left(\bar{x}_{k}\right)=z_{k}^{T} \Omega_{1} z_{k}-r_{1} \bar{x}_{k}^{T} P^{-1} \bar{x}_{k}+r_{1} w_{k}^{T} w_{k},
$$

where

$$
\Omega_{1}=\left[\begin{array}{cc}
A_{c l}^{T} P^{-1} A_{c l}-P^{-1}+r_{1} P^{-1} & A_{c l}^{T} P^{-1} \mathcal{H} \\
* & \mathcal{H}^{T} P^{-1} \mathcal{H}-r_{1} I
\end{array}\right] .
$$

Hence, since $w_{\infty}^{2} \geq w_{k}^{T} w_{k}$ for all $k \geq 0$, if $\Omega_{1} \preceq 0, \Delta V_{1}\left(\bar{x}_{k}\right) \leq r_{1}\left(w_{\infty}^{2}-V_{1}\left(\bar{x}_{k}\right)\right)$, where the latter inequality ensures that any closed-loop system trajectory outside of the ellipsoid $V_{1}\left(\bar{x}_{k}\right)=w_{\infty}^{2}$ will converge to itself as $k$ goes to infinity. On the other hand negativity of $(1,1)$ entry of $\Omega_{1}$ automatically ensures the invariance of $\mathcal{E}\left(P^{-1}, w_{\infty}^{2}\right)$. Therefore, the region defined by the ellipsoid

$$
\mathcal{E}\left(P^{-1}, w_{\infty}^{2}\right) \triangleq\left\{\bar{x}_{k} \mid \bar{x}_{k}^{T} P^{-1} \bar{x}_{k} \leq w_{\infty}^{2}\right\},
$$


is attractive and invariant for the closed-loop system (9). Note that, using Schur complement formula [33] on (13), the condition $\Omega_{1} \preceq 0$ is equivalent to

$$
\left[\begin{array}{ccc}
\left(r_{1}-1\right) P^{-1} & 0 & A_{c l}^{T} P^{-1} \\
* & -r_{1} I & \mathcal{H}^{T} P^{-1} \\
* & * & -P^{-1}
\end{array}\right] \preceq 0 .
$$

the modified condition

$$
\bar{x}_{k}^{T} \ell_{i} \frac{w_{\infty}^{2}}{\bar{u}_{i-n}^{2}} \ell_{i}^{T} \bar{x}_{k} \leq w_{\infty}^{2}, \quad i=n+1, \ldots, n+m, \forall k \geq 0 .
$$

Since any closed-loop system trajectory starting inside the ellipsoid $\mathcal{E}\left(P^{-1}, w_{\infty}^{2}\right)$ can never leave it, satisfaction of

$$
\bar{x}_{k}^{T} \ell_{i} \frac{w_{\infty}^{2}}{\bar{u}_{i-n}^{2}} \ell_{i}^{T} \bar{x}_{k} \leq \bar{x}_{k}^{T} P^{-1} \bar{x}_{k}, \quad i=n+1, \ldots, n+m, \forall k \geq 0,
$$

automatically ensures the feasibility of $(19)$ which is further equivalent to

$$
P^{-1}-\ell_{i}\left(\frac{w_{\infty}^{2}}{\bar{u}_{i-n}^{2}}\right) \ell_{i}^{T} \geq 0, \quad i=n+1, \ldots, n+m, \forall k \geq 0 .
$$

Finally, applying a congruence transformation on (21) by using $P=P^{T} \succeq 0$ and the Schur complement formula, (21) can be represented by the LMI (7).

Apart from the magnitude constraint, the control signal should also satisfy the slew-rate constraint $\left|v_{i, k}\right| \leq \bar{v}_{i}, \forall k \geq 0, i=1, \ldots, m$, which is nothing but equivalent to

$$
\left|v_{i, k}\right|^{2}=\bar{x}_{k}^{T} K_{i}^{T} K_{i} \bar{x}_{k}=\bar{x}_{k}^{T} G^{-T} L^{T} \ell_{i} \ell_{i}^{T} L G^{-1} \bar{x}_{k} \leq \bar{v}_{i}^{2}, \quad \forall k \geq 0, i=1, \ldots, m .
$$


Similar to the bounding condition on the magnitude constraints, the rate of signal is upper bounded by $\bar{v}_{i}$ if

$$
\bar{x}_{k}^{T} G^{-T} L^{T} \ell_{i}\left(\frac{w_{\infty}}{\bar{v}_{i}}\right)^{2} \ell_{i}^{T} L G^{-1} \bar{x}_{k} \leq w_{\infty}^{2} .
$$

Note that using congruence transformation, this condition is satisfied if

$$
G^{T} P^{-1} G \succeq L^{T} \ell_{i}\left(\frac{w_{\infty}}{\bar{v}_{i}}\right)^{2} \ell_{i}^{T} L \quad i=1, \ldots, m .
$$

Then, in light of the property (17) and the Schur complement formula, (24) is equivalent to (6). This concludes the proof.

Remark 1. Our task is to reduce the effects of disturbances $w_{k}$ on the state ector $x_{k}$ as $k$ goes to infinity. One way volume of the invariant ellipsoid $\mathcal{E}\left(P^{-1}, w_{\infty}^{2}\right)$ as much as possible. It is well known that for a fixed value of $w_{\infty}$, the volume of the ellipsoid $\mathcal{E}\left(P^{-1}, w_{\infty}^{2}\right)$ is directly proportional to Trace $(P)$. Hence, one can solve the following optimisation problem in order to find the optimal controller strategy to reduce the effects of disturbances, $w$ on states $x$ :

$$
\begin{aligned}
& \text { minimise } \operatorname{Trace}(P) \\
& \text { subject to }
\end{aligned}
$$

Remark 2. To obtain a minimum achievable Trace $(P)$ which satisfies (5), one needs to perform a line search over $r_{1}$ (which is in fact introduced to facilitate a reduction in conservatism).

Using the control method proposed in Theorem 1, the closed-loop system can tions are inside the invariant set $\mathcal{E}\left(P^{-1}, w_{\infty}^{2}\right)$. On the other hand, the optimisation problem 25 seeks a minimum volume ellipsoid as time goes to infinity which might cause the actuators to saturate for almost every initial condition except the ones that start from rest or very close to the origin.

To this end, we shall slightly modify Theorem 1 to introduce another maximal invariant ellipsoid $\mathcal{E}\left(Q^{-1}, w_{\infty}^{2}\right)$ inscribed by the polyhedral region defined by the linear control constraints $\sqrt{2}$ which encloses the attractive invariant ellipsoid $\mathcal{E}\left(P^{-1}, w_{\infty}^{2}\right)$ at all times. This guarantees that all closed-loop system trajectories that start within the outer ellipsoid converge to the inner one in a finite time without violating the control constraints (2).

Theorem 2. For given scalars $r_{\{1,2\}} \in[0,1]$, there exists a structured stabilizing controller of the form (4) for nominal system (1) that is subject to the constraints 2), if there exist $P=P^{T} \in \mathbb{R}^{(n+m) \times(n+m)} \succ 0, Q=Q^{T} \in$ $\mathbb{R}^{(n+m) \times(n+m)} \succ 0, G \in \mathbb{R}^{(n+m) \times(n+m)}$ and $L \in \mathbb{R}^{m \times(n+m)}$ which satisfy the 245 following linear matrix inequalities:

$$
\left[\begin{array}{ccc}
\left(1-r_{1}\right)\left(G+G^{T}-P\right) & 0 & G^{T} \mathcal{A}^{T}+L^{T} \mathcal{B}^{T} \\
* & r_{1} I & \mathcal{H}^{T} \\
* & * & P
\end{array}\right] \succeq 0
$$




$$
\begin{aligned}
& {\left[\begin{array}{ccc}
r_{2}\left(G+G^{T}-Q\right) & 0 & G^{T} \mathcal{A}^{T}+L^{T} \mathcal{B}^{T} \\
* & \left(1-r_{2}\right) I & \mathcal{H}^{T} \\
* & * & Q
\end{array}\right] \succeq 0,} \\
& {\left[\begin{array}{cc}
G+G^{T}-Q & L^{T} \ell_{i}^{T} \\
\ell_{i} L & \left(\frac{\bar{v}_{i}}{w_{\infty}}\right)^{2}
\end{array}\right] \succeq 0, \quad i=1, \ldots, m,} \\
& {\left[\begin{array}{cc}
Q & Q \ell_{i} \\
\ell_{i}^{T} Q & \left(\frac{\bar{u}_{i-n}}{w_{\infty}}\right)^{2}
\end{array}\right] \succeq 0, \quad i=n+1, \ldots, n+m,} \\
& Q-P \succeq 0 .
\end{aligned}
$$

250 If a solution exists to the inequalities above, then $\bar{x}_{k}^{T} P^{-1} \bar{x}_{k} \leq w_{\infty}^{2}$ is a state-invariant attractive ellipsoid nested inside the outer invariant ellipsoid $\bar{x}_{k}^{T} Q^{-1} \bar{x}_{k} \leq w_{\infty}^{2}$ and the stabilizing controller gain is given by $K=$ $L G^{-1}$. Hence, any closed-loop system trajectory having initial conditions inside $\mathcal{E}\left(Q^{-1}, w_{\infty}^{2}\right)$ will converge to $\mathcal{E}\left(P^{-1}, w_{\infty}^{2}\right)$ with a control action that satisfies (2).

255 Proof 2. We have already shown in the proof of Theorem 1 that (26) implies the invariance and attractiveness of the ellipsoid $\mathcal{E}\left(P^{-1}, w_{\infty}^{2}\right)$. To show that a feasible solution to (27) guarantees the invariance of $\mathcal{E}\left(Q^{-1}, w_{\infty}^{2}\right)$, consider another energy function of the form $V_{2}\left(\bar{x}_{k}\right) \triangleq \bar{x}_{k}^{T} Q^{-1} \bar{x}_{k}, Q=Q^{T} \succ 0$ which satisfies

$$
V_{2}\left(\bar{x}_{k+1}\right)-r_{2} V_{2}\left(\bar{x}_{k}\right)-\left(1-r_{2}\right) w_{k}^{T} w_{k} \leq 0, \forall k \geq 0,
$$

along the closed-loop system trajectory (9). Since $w_{k}^{T} w_{k} \leq w_{\infty}^{2}$ for all $k \geq 0$, one can always write

$$
V_{2}\left(\bar{x}_{k+1}\right) \leq r_{2} V_{2}\left(\bar{x}_{k}\right)+\left(1-r_{2}\right) w_{\infty}^{2},
$$

from (31). Now, taking an arbitrary state trajectory $\bar{x}_{k}$ within the ellipsoid $\mathcal{E}\left(Q^{-1}, w_{\infty}^{2}\right)$ yields $\bar{x}_{k}$ to satisfy $V_{2}\left(\bar{x}_{k+1}\right) \leq w_{\infty}^{2}$ for all $k \geq 0$ since $r_{2} \in[0,1]$. This guarantees that any trajectory within the ellipsoid $\mathcal{E}\left(Q^{-1}, w_{\infty}^{2}\right)$ will never leave it. Hence, using closed-loop system trajectory (9) in (32) yields

$$
\left[\begin{array}{c}
\bar{x}_{k} \\
w_{k}
\end{array}\right]^{T} \underbrace{\left[\begin{array}{cc}
A_{c l}^{T} Q^{-1} A_{c l}-r_{2} Q^{-1} & A_{c l}^{T} Q^{-1} \mathcal{H} \\
* & \mathcal{H}^{T} Q^{-1} \mathcal{H}+\left(r_{2}-1\right) I
\end{array}\right]}_{\Omega_{2}}\left[\begin{array}{c}
\bar{x}_{k} \\
w_{k}
\end{array}\right] \leq 0 .
$$

Using Schur complement formula on (33), $\Omega_{2} \preceq 0$ is equivalent to

$$
\left[\begin{array}{ccc}
-r_{2} Q^{-1} & 0 & A_{c l}^{T} Q^{-1} \\
* & \left(r_{2}-1\right) I & \mathcal{H}^{T} Q^{-1} \\
* & * & -Q^{-1}
\end{array}\right] \preceq 0 .
$$

Then, replacing $A_{c l}$ with $\mathcal{A}+\mathcal{B} K, L$ with $K G$ in (34), and using the fact that $-G^{T} Q^{-1} Q \preceq Q-G-G^{T} \prec 0$, one can apply a congruence transformation 
on (34) with the transformation matrix $\operatorname{diag}\{G, I, Q\}$ to obtain (27). On the other hand, (30) ensures that $\mathcal{E}\left(P^{-1}, w_{\infty}^{2}\right) \subseteq \mathcal{E}\left(Q^{-1}, w_{\infty}^{2}\right)$ at all times. Finally, following a similar procedure carried out in the proof of Theorem 1, it can be shown that a feasible solution to (28) and (29) ensures that the polyhedra defined by the the inequalities (2) encapsulates $\mathcal{E}\left(Q^{-1}, w_{\infty}^{2}\right)$. This concludes the proof.

to obtain the maximum volume ellipsoid almost perfectly. However, not all numerical solvers in the market support the maxdet problem. Therefore, in the sequel, an alternative approach is recommended at the cost of some added conservatism.

Note that, it follows from arithmetic mean-geometric mean inequality 35 that

$$
\left(\operatorname{det}\left(Q^{-1}\right)\right)^{1 / 2} \leq \frac{\operatorname{Trace}\left(Q^{-1}\right)}{2},
$$

which implies that

$$
\operatorname{Vol} \mathcal{E}\left(Q^{-1}, w_{\infty}^{2}\right) \geq \frac{2 t_{n}}{\operatorname{Trace}\left(Q^{-1}\right)}
$$

Therefore, to maximise the volume of $\mathcal{E}\left(Q^{-1}, w_{\infty}^{2}\right)$, one needs to minimise Trace $\left(Q^{-1}\right)$. To this end, we introduce a new matrix $S=S^{T} \succ 0$ with the 300 same size of $Q$ such that

$$
\text { Trace }\left(Q^{-1}\right) \leq \operatorname{Trace}(S),
$$


which is equivalent to the LMI condition

$$
\left[\begin{array}{ll}
S & I \\
I & Q
\end{array}\right] \succeq 0
$$

Now, we have all ingredients to propose the following optimisation problem to obtain the best control design depending on the needs of the closed-loop control system:

$$
\begin{array}{ll}
\underset{\lambda \in[0,1]}{\operatorname{minimise}} & \lambda \operatorname{Trace}(P)+(1-\lambda) \operatorname{Trace}(S) \\
\text { subject to } & 26,27,28,29,230) \text { and } 239
\end{array}
$$

Remark 3. The sufficient conditions that are obtained in Theorem 1 and then in Theorem 2 are non-convex unless $r_{1}$ and $r_{2}$ are fixed values. To find nearoptimal values of $r_{1}$ and $r_{2}$, one can perform gridding on the $r_{1}-r_{2}$ plane with a sufficiently fine resolution $\left(d r_{1}, d r_{2}\right)$. On the other hand, since $\operatorname{Trace}(P)$ and $\operatorname{Trace}(S)$ are conflicting objectives, the contribution of each constraint to the cost function is weighted by a tuning parameter $\lambda \in[0,1]$. This weight may be adjusted to achieve a desired trade-off between each component in the cost function. If all initial conditions are set to zero then focusing only on the minimisation of $\operatorname{Trace}(P)$ ( $\lambda$ close to 1) allows the user to get perfect regulation.

315 However, if one or more of the initial conditions are non-zero, then the user may slide the objective towards $\operatorname{Trace}(S)$ by decreasing $\lambda$ until an acceptable response is achieved.

\section{Robust Controller Synthesis}

Now let us consider the case in which the extended plant is uncertain or having time varying parameters. We represent the plant by Linear Fractional Transformation (LFT) as follows:

$$
\left[\mathcal{A}\left(\Delta_{k}\right)\left|\mathcal{B}\left(\Delta_{k}\right)\right| \mathcal{H}\left(\Delta_{k}\right)\right]=[\mathcal{A}|\mathcal{B}| \mathcal{H}]+\mathcal{B}_{p} \Delta_{k}\left[I-\mathcal{D}_{p} \Delta_{k}\right]^{-1}\left[\mathcal{A}_{d}\left|\mathcal{B}_{d}\right| \mathcal{H}_{d}\right]
$$

where $\mathcal{A}_{d}, \mathcal{B}_{d}, \mathcal{H}_{d}, \mathcal{B}_{p}$ and $\mathcal{D}_{p}$ are known system matrices of appropriate dimensions. The system matrices are assumed to depend rationally on an unknown (possibly) time-varying parameter matrix $\Delta_{k}$ (hereinafter referred to as

${ }_{325} \Delta$ ) which satisfies $\Delta \in \Delta, \forall k \geq 0$ where $\boldsymbol{\Delta}$ is a compact uncertainty set. For obtaining the LFT representation of a given uncertain system, one can refer to [36] or 37. Note that since we employ full block S-procedure, our method neither requires a particular structure in $\boldsymbol{\Delta}$ nor needs to explicitly check for the well-posedness of the LFT representation.

${ }_{330}$ To derive robust synthesis conditions which are tractable and less conservative, we employ full block S-procedure [31] to our problem. The following Lemma is crucial for us to provide a robust synthesis solution to the problem stated in Section 2 for systems having uncertain system matrices given in 41). 
However, as an ingredient, let us first define a multiplier matrix $\Phi$ which satisfies

335

$$
\left[\begin{array}{c}
\Delta^{T} \\
I
\end{array}\right]^{T} \underbrace{\left[\begin{array}{cc}
\Psi & \chi \\
\chi^{T} & \Pi
\end{array}\right]}_{\Phi}\left[\begin{array}{c}
\Delta^{T} \\
I
\end{array}\right]=\Delta \Psi \Delta^{T}+\operatorname{He}\{\Delta \chi\}+\Pi \preceq 0, \forall \Delta \in \Delta .
$$

Also, let us introduce the LFT

$$
\Delta \star \underbrace{\left[\begin{array}{l|l}
\mathcal{Y}_{11} & \mathcal{Y}_{12} \\
\hline \mathcal{Y}_{21} & \mathcal{Y}_{22}
\end{array}\right]}_{\mathcal{Y}} \triangleq \mathcal{Y}_{22}+\mathcal{Y}_{21} \Delta\left(I-\mathcal{Y}_{11} \Delta\right)^{-1} \mathcal{Y}_{12}
$$

which is said to be well-posed when $\left(I-\mathcal{Y}_{11} \Delta\right)$ is invertible for all $\Delta \in \Delta$.

Lemma 1. [38] The LFT $\Delta \star \mathcal{Y}$ is well-posed and

$$
\operatorname{He}\{\Delta \star \mathcal{Y}\} \triangleq \Delta \star \mathcal{Y}+(\Delta \star \mathcal{Y})^{T} \succeq 0, \Delta \in \Delta,
$$

holds if and only if there exists a matrix $\Phi$ that satisfies (42) and

$$
\left[\begin{array}{cc}
\mathcal{Y}_{21} \Pi \mathcal{Y}_{21}^{T}+\operatorname{He}\left\{\mathcal{Y}_{22}\right\} & \mathcal{Y}_{21} \Pi \mathcal{Y}_{11}^{T}+\mathcal{Y}_{21} \chi^{T}+\mathcal{Y}_{12}^{T} \\
* & \Psi+\mathcal{Y}_{11} \Pi \mathcal{Y}_{11}^{T}+\operatorname{He}\left\{\mathcal{Y}_{11} \chi^{T}\right\}
\end{array}\right] \succeq 0
$$

340 Now it is possible for us to establish the following result:

Theorem 3. Consider system (1) together with constraints in (2) and the augmented system (3) along with the uncertain system matrices (41). Given the scalars $r_{1,2} \in[0,1]$, there exists a robust controller of the form

$$
u_{k}=u_{k-1}+K\left[\begin{array}{ll}
x_{k}^{T} & u_{k-1}^{T}
\end{array}\right]^{T},
$$

if there exist matrices $P=P^{T} \in \mathbb{R}^{(n+m) \times(n+m)} \succ 0, Q=Q^{T} \in$

the infinite dimensional matrix inequality (42), LMIs (28), (29), (30) and the following:

$$
\begin{gathered}
{\left[\begin{array}{cccc}
\left(1-r_{1}\right)\left(G+G^{T}-P\right) & 0 & * & * \\
0 & r_{1} I & * & * \\
\mathcal{A} G+\mathcal{B} L & \mathcal{H} & P+\mathcal{B}_{p} \Pi \mathcal{B}_{p}^{T} & * \\
\mathcal{A}_{d} G+\mathcal{B}_{d} L & \mathcal{H}_{d} & \mathcal{D}_{p} \Pi \mathcal{B}_{p}^{T}+\chi^{T} & \Sigma
\end{array}\right] \succeq 0,} \\
{\left[\begin{array}{cccc}
r_{2}\left(G+G^{T}-Q\right) & 0 & * & * \\
0 & \left(1-r_{2}\right) I & * & * \\
\mathcal{A} G+\mathcal{B} L & \mathcal{H} & Q+\mathcal{B}_{p} \Pi \mathcal{B}_{p}^{T} & * \\
\mathcal{A}_{d} G+\mathcal{B}_{d} L & \mathcal{H}_{d} & \mathcal{D}_{p} \Pi \mathcal{B}_{p}^{T}+\chi \mathcal{B}_{p}^{T} & \Sigma
\end{array}\right] \succeq 0,}
\end{gathered}
$$

where $\Sigma=\Psi+\mathcal{D}_{p} \Pi \mathcal{D}_{p}^{T}+\operatorname{He}\left\{\mathcal{D}_{p} \chi^{T}\right\}$. If a feasible solution can be obtained to the LMIs above, $\mathcal{E}\left(P^{-1}, w_{\infty}^{2}\right)$ will be a state-invariant, robustly attractive ellipsoid nested inside the larger ellipsoid $\mathcal{E}\left(Q^{-1}, w_{\infty}^{2}\right)$ and the robust controller gain is given by $K=L G^{-1}$. Also, any closed-loop system trajectory having initial conditions inside $\mathcal{E}\left(Q^{-1}, w_{\infty}^{2}\right)$ will converge to the inner ellipsoid $\mathcal{E}\left(P^{-1}, w_{\infty}^{2}\right)$ with a control action that satisfies (2). 
Proof 3. Only changes in the proof of Theorem 2 are on the conditions which involve system matrices. Using the uncertain matrix definitions (41), the matrix inequality (26) can be rewritten as

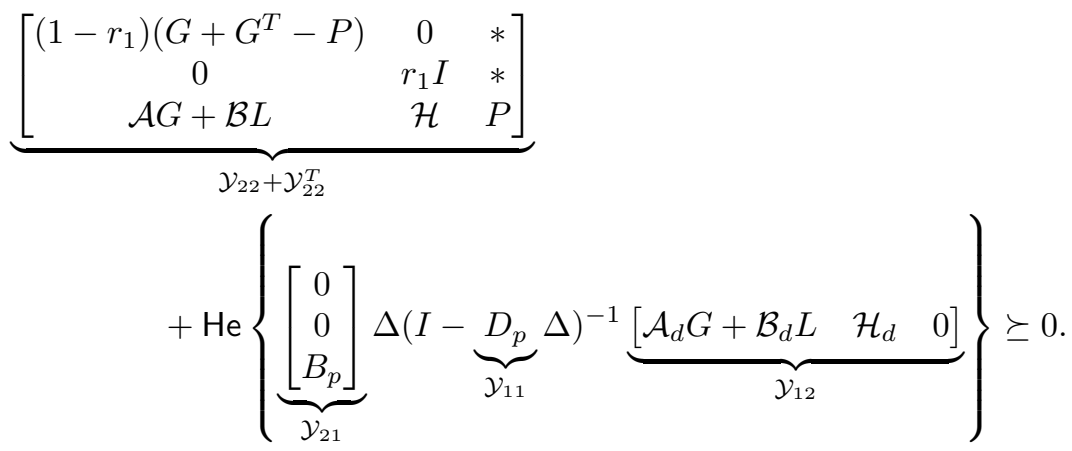

Then, (49), is equivalent to (47) in light of Lemma 1. By following a similar procedure, (27) can be replaced by (48). This concludes the proof.

We note that $(42)$ has to be satisfied for all admissible values of $\Delta \in \Delta$. However, this requires a feasibility check of infinitely many LMI conditions. Hence, one needs to obtain tractable sufficient conditions instead of 444. To this end, we need to employ some relaxation methods to derive finitely many LMI conditions that ensure (42). For polytopic regions, convex-hull relaxation or Pólya's method can be used. However, for regions described by polynomial inequalities, sum-of-squares (SOS) approach would be much more suitable (see 39 and 40] and the references therein). In the next subsection we summarize two relaxation methods namely the convex-hull relaxation and Pólya relaxation 41 to check the feasibility of (42) by means of a finite number of LMI constraints.

\subsection{Relaxations for Polytopic Regions}

A polytopic uncertainty region can be described as the convex-hull of a given set of finitely many matrices $\boldsymbol{\Delta}_{e}=\left\{\Delta^{1}, \ldots, \Delta^{\kappa}\right\}$ as follows:

$$
\boldsymbol{\Delta}=\operatorname{conv}\left(\boldsymbol{\Delta}_{e}\right) \triangleq\left\{\sum_{j=1}^{\kappa} \alpha_{j} \Delta^{j}: \sum_{j=1}^{\kappa} \alpha_{j}=1, \alpha_{j} \geq 0\right\}
$$

Before we discuss the suitable relaxations for polytopic regions, we first introduce the following notation:

$$
\Gamma_{i j}(\Phi) \triangleq\left[\begin{array}{c}
\left(\Delta^{i}\right)^{T} \\
I
\end{array}\right]^{T} \Phi\left[\begin{array}{c}
\left(\Delta^{j}\right)^{T} \\
I
\end{array}\right], i, j=1, \ldots, \kappa .
$$

\subsubsection{Convex-Hull Relaxation}

In this approach, we first ensure that the left hand side of 442 is a convex function of $\Delta$. It then becomes possible to ensure 42 for all $\Delta \in \Delta$ simply by 
enforcing the condition only at the extreme points $\Delta^{j}$. The convexity condition read as

$$
\begin{aligned}
& \left\{a\left[\begin{array}{c}
\left(\Delta^{1}\right)^{T} \\
I
\end{array}\right]^{T}\left[\begin{array}{cc}
\Psi & \chi \\
\chi^{T} & \Pi
\end{array}\right]\left[\begin{array}{c}
\left(\Delta^{1}\right)^{T} \\
I
\end{array}\right]+(1-a)\left[\begin{array}{c}
\left(\Delta^{2}\right)^{T} \\
I
\end{array}\right]^{T}\left[\begin{array}{cc}
\Psi & \chi \\
\chi^{T} & \Pi
\end{array}\right]\left[\begin{array}{c}
\left(\Delta^{2}\right)^{T} \\
I
\end{array}\right]\right. \\
& \left.\succeq\left(a \Delta^{1}+(1-a) \Delta^{2}\right) \Psi\left(a \Delta^{1}+(1-a) \Delta^{2}\right)^{T}+\operatorname{He}\left\{a \Delta^{1} \chi+(1-a) \Delta^{2} \chi\right\}+\Pi\right\} \\
& \Leftrightarrow a(1-a)\left(\Delta^{1}-\Delta^{2}\right) \Psi\left(\Delta^{1}-\Delta^{2}\right)^{T} \succcurlyeq 0, \forall a \in[0,1], \forall \Delta^{1}, \Delta^{2} \in \Delta \text {. }
\end{aligned}
$$

A sufficient condition is observed easily from this expression as $\Psi \succcurlyeq 0$. In conclusion, the convex hull relaxation is identified by the following conditions:

$$
\begin{aligned}
\Psi & \succcurlyeq 0, \\
\Gamma_{j j}(\Phi) & \preccurlyeq 0, \quad j=1, \ldots, \kappa
\end{aligned}
$$

Remark 4. In the case of affine parameter dependence, i.e. $\mathcal{Y}_{11}=0$, it is already implied by (45) that $\Psi \succeq 0$. It would hence be enough to impose (42) only at the extreme points of the uncertainty region in the case of affine parameter dependence.

\subsubsection{Relaxation based on Pólya's Method}

380 The idea behind the Pólya approach is to express the left hand side of 42 ) as a polynomial matrix that is homogeneous in $\alpha$. In the simplest case, we can achieve this with a polynomial order of two:

$$
\sum_{j=1}^{\kappa} \alpha_{j}^{2} \Gamma_{j j}+\sum_{j=1}^{\kappa} \sum_{i=j+1}^{\kappa} \alpha_{j} \alpha_{i}\left(\Gamma_{j i}+\Gamma_{j i}^{T}\right) \preccurlyeq 0 .
$$

With the selection of $\alpha_{j} \in[0,1]$, one can state a set of sufficient LMI conditions for 42 as follows:

$$
\begin{aligned}
\Gamma_{j j}(\Phi) & \preccurlyeq 0, \quad j=1, \ldots, \kappa \\
\operatorname{He}\left(\Gamma_{j i}(\Phi)\right) & \preccurlyeq \quad 0, \quad j=1, \ldots, \kappa ; i=j+1, \ldots, \kappa
\end{aligned} .
$$

For details, please refer to [40] and 38].

\section{Robust gain scheduling control}

The controller techniques introduced in the previous sections are based on the knowledge of the worst case(peak) disturbance $w_{\infty}$ which unlikely occur in most cases. Therefore, using fixed controller gains that are generated based on the worst case disturbance scenario would be over conservative and may lead to a large surrounding ellipsoid producing small controller gains with a degradation in the performance. One approach to overcome this issue would be generating $N>1 \in \mathbb{Z}$ intermediate controllers using different levels of disturbances predefined in an interval $\left[w_{\min }<\cdots<w_{i}<w_{i+1}<\cdots<w_{\infty}\right]$ and switching between 


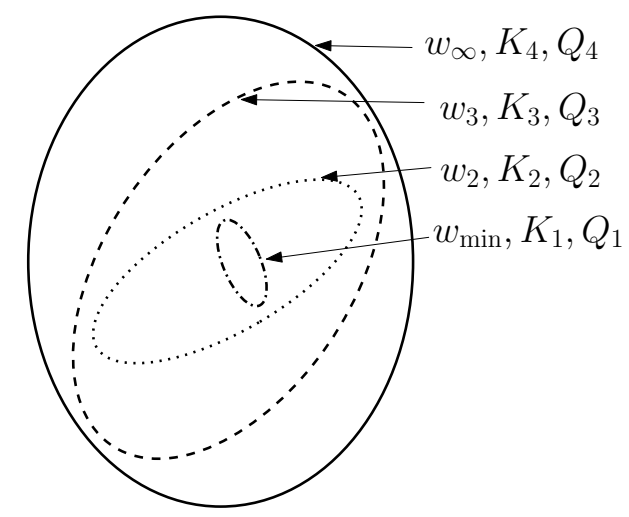

Figure 1: The ellipsoids associated with the gain-scheduling controllers $(N=4)$.

395 them during the online operation whenever its is necessary. It is obvious that while the controller $K_{1}$ designed for $w_{\min }$ is the most aggressive one having the smallest ellipsoid and therefore the highest controller gains, the controller $K_{N}$ that is designed for $w_{\infty}$ is the safest with the largest ellipsoid and never saturates for any disturbance within the bounds. Therefore, the proposed gain400 scheduling controller with a highest gain (smaller $i$ ) that is not saturated would be the one to be used at all times. If the amplitude of disturbance increases at any time and the corresponding $K_{i}$ saturates, the gain-scheduling mechanism switches to the following available lower gain controller (by increasing $i$ ) that is not saturated, until it reaches $K_{N}$. In contrary, if at any time there is 405 more aggressive controller that is not saturated, the gain-scheduling mechanism switches back to it. This approach guarantees the use of the most aggressive controller available at any time. Similar idea has been used in the literature [42, [26], 27] for different continuous-time control problems having different objective functions. However, thanks to the velocity form description introduced in this paper, the aggressive switching issue between the $N$ controllers mentioned in 42 is not a problem with our technique since our method manipulates the rate of the control signal rather than the control signal itself.

On the other hand, to achieve a safe switching between controllers that guarantees the closed-loop stability at all times, the off-line controller design process must ensure that $Q_{i+1} \succeq Q_{i}$ for all $i=1, \ldots, N-1$. The situation is demonstrated in Figure 1 for $N=4$ case. The overall design method is summarized in the Algorithm 1. The algorithm requires the determination of the parameters $w_{\min }$ and the integer $N$. Although a trivial selection might be selecting $w_{\min }$ close to zero, there always be an effective value of $w_{\min }>0$ for 420 every system and the controllers designed for all disturbance limits below this value have almost the same controller gains. One approach to identify this value would be checking the norm of the controller gains by reducing the lower limit $w_{\text {min }}$ gradually until observing almost no change in the controller gain. 


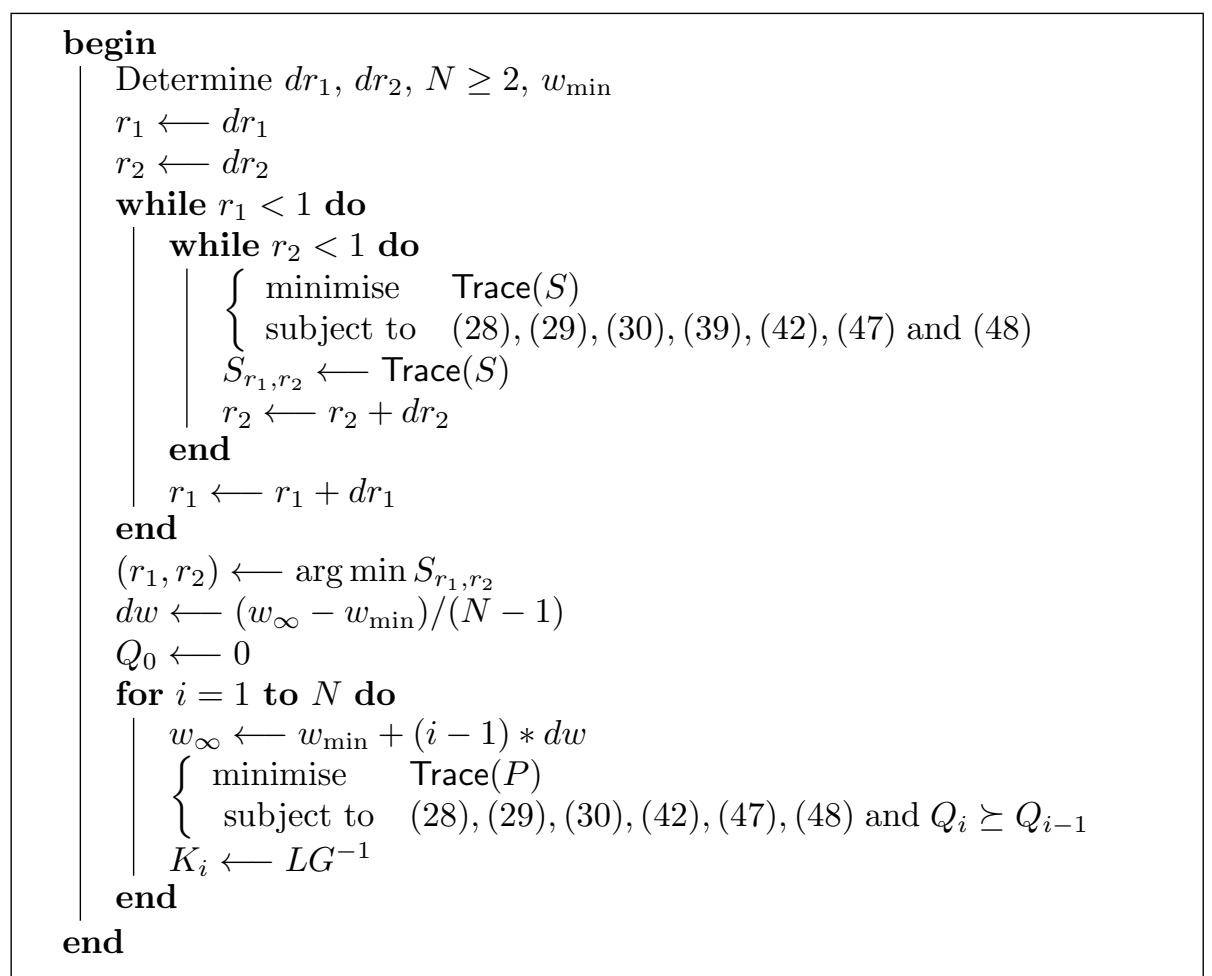

Algorithm 1: Summary of the design process for the robust gainscheduling controller.

Once a family of $N$ controller gains are obtained off-line using the Algorithm 1, the gain-scheduling mechanism can be implemented in real time according to the rule base shown in Algorithm 2

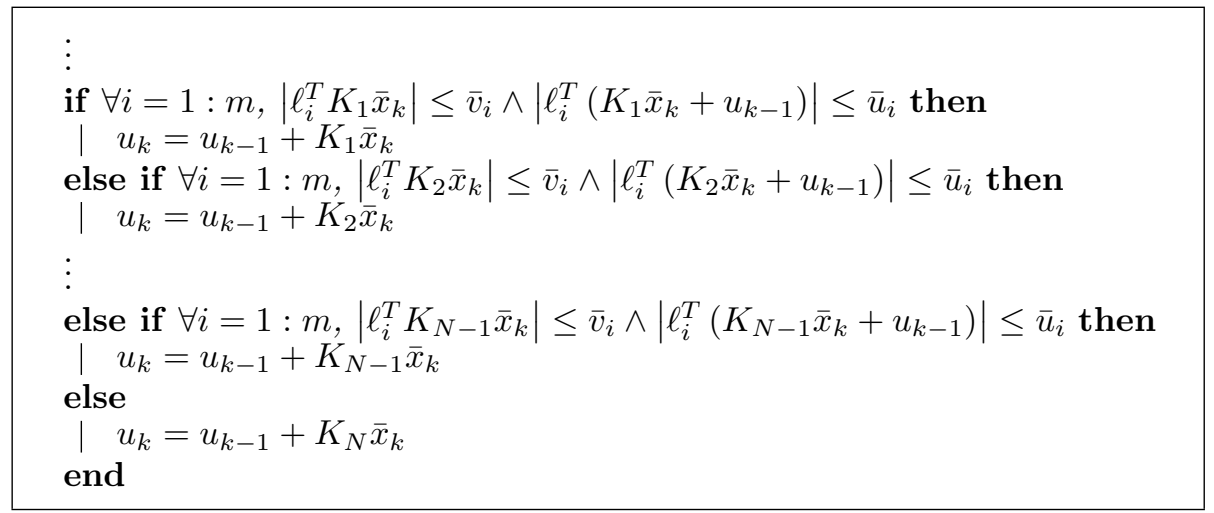

Algorithm 2: Implementation of the proposed gain-scheduling controller. 


\section{Simulation results}

${ }_{430}$ In this section, we apply the synthesis procedure to the linearised model of a damped inverted pendulum which is actuated by an actuator having a first order dynamics of the form $G_{a}(s)=\frac{\tau}{s+\tau}$. Using Lagrangian modelling and Euler discretization, we obtain the nominal discrete-time state-space model of the pendulum system as follows:

$$
\left[\begin{array}{l}
x_{1}(k+1) \\
x_{2}(k+1) \\
x_{3}(k+1)
\end{array}\right]=\left[\begin{array}{ccc}
1 & T_{s} & 0 \\
\frac{g T_{s}}{l} & 1-\frac{c T_{s}}{m l^{2}} & \frac{T_{s}}{m l^{2}} \\
0 & 0 & 1-\tau T_{s}
\end{array}\right]\left[\begin{array}{l}
x_{1}(k) \\
x_{2}(k) \\
x_{3}(k)
\end{array}\right]+\left[\begin{array}{c}
0 \\
0 \\
\tau T_{s}
\end{array}\right] u(k)+\left[\begin{array}{c}
0 \\
\frac{T_{s}}{m l^{2}} \\
0
\end{array}\right] w(k),
$$

where $x_{1}[\mathrm{rad}]$ is the angle of the pendulum with respect to vertical axis, $x_{2}[\mathrm{rad} / \mathrm{s}]$ is the angular velocity of the pendulum, $x_{3}$ is the state of the actuator. The sampling period is chosen as $T_{s}=0.01 \mathrm{~s} . g=9.8 \mathrm{~m} / \mathrm{s}^{2}$ is the acceleration of gravity whereas $m=1 \mathrm{~kg}$ is the mass of the pendulum. $l=1 \mathrm{~m}$ stands for the length of the pendulum and $c=0.1 \mathrm{Ns} / \mathrm{m}$ denotes the damping coefficient of the damper. Finally, $1 / \tau=0.1$ is the time constant of the actuator. Similar problem with normalised parameters has been considered in [27] and [26]. All simulations are implemented in MATLAB, using the parser YALMIP [43] and LMI Lab solver on a $2.7 \mathrm{GHz}$ core-i7 computer having 16 GB RAM running MacOS.

445 Four different scenarios are considered during demonstrations. As a first case study, nominal controller is implemented on the system model having constant parameters at their nominal values. Then the proposed gain-scheduling controller is applied to the nominal system as the second case study. As a third case, the performance of the proposed robust controller is demonstrated on the system having uncertain parameters $m, c$ and $\tau$. Finally, as a last case, robust gain-scheduling controller's performance is evaluated on the uncertain time-varying plant. In entire study, it is assumed that all initial conditions are zero and the system is subject to a disturbance

$$
w(t)= \begin{cases}-2.5 & 0 \leq t \leq 1.25 \\ 2.5 \operatorname{sign}(\sin (0.5 \pi t)) & 1.25<t \leq 3.33 \\ 0 & \text { otherwise }\end{cases}
$$

which lead to a worst case disturbance $w_{\infty}=2.5$. Based on the restrictions 455 imposed by the actuator dynamics, magnitude and rate bounds are $\bar{u}=10$ and $\bar{v}=1$, respectively. Finally, to evaluate the performance of the proposed controllers, all controllers are compared with the linear quadratic regulator(LQR) having a control law $u_{L Q R}(k)=K_{L Q R} x(k)$ with a gain matrix $K_{L Q R}=[-25.3648-8.0256-0.6129]$ obtained by utilising the cost function

$$
J_{L Q R}=\sum_{k=0}^{\infty} x^{T}(k)\left[\begin{array}{ccc}
10 & 0 & 0 \\
0 & 10 & 0 \\
0 & 0 & 0.01
\end{array}\right] x(k)+10 u_{L Q R}^{2}(k)
$$



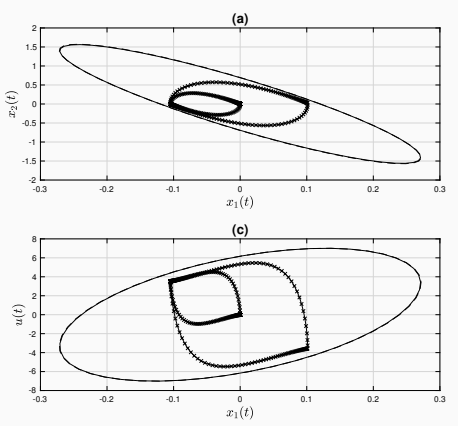

(e)

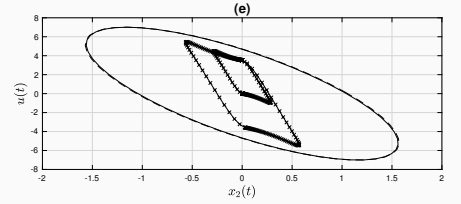

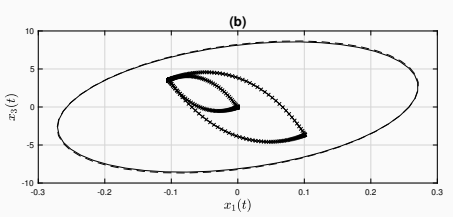

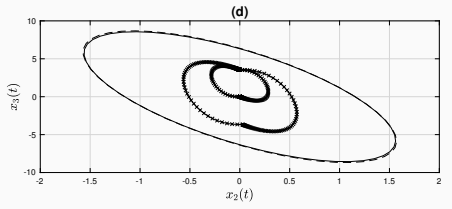

(f)

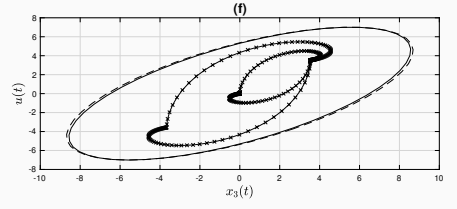

Figure 2: (Nominal case) Projection of the closed-loop system trajectories $(-\mathrm{x})$ together with the invariant ellipsoids $\mathcal{E}\left(P^{-1}, w_{\infty}^{2}\right)$ (solid) and $\mathcal{E}\left(Q^{-1}, w_{\infty}^{2}\right)$ (dashed) on the corresponding states.

The weights of the cost function are determined in such a way that the controller can focus only on the regulation of the states $x_{1}$ and $x_{2}$ without violating the control signal bounds.

In case 1, the implementation of Algorithm 1 leads to the design parameters $r_{1}=0.1$ and $r_{2}=0.9$ and the controller gain

$$
K=\left[\begin{array}{llll}
-26.4214 & -6.7358 & -0.4560 & -0.3289
\end{array}\right] .
$$

465 Figure 2 shows the projections of the trajectories together with the bounding ellipsoids. Since all initial conditions are taken as zero, by taking $\lambda=1$, the controller design is fully focused on the disturbance rejection rather than maximisation of the region of attraction. Therefore, the ellipsoid $\mathcal{E}\left(Q^{-1}, w_{\infty}^{2}\right)$ almost fully overlaps with $\mathcal{E}\left(P^{-1}, w_{\infty}^{2}\right)$. Figure 3 shows the variation of the control signals both for the proposed controller and the LQR. It is appendant that both controllers do not generate control signals that violate the magnitude and rate bounds of the system. The closed-loop state trajectories for the proposed controller and the LQR are shown in Figure 4. It is obvious that the proposed controller outperforms LQR for the attenuation of disturbance signal on the 475 states $x_{1}$ and $x_{2}$ by significantly reducing the oscillations of the states. Note that the variation of the state vector $x_{3}$ is not demonstrated as it stands for the state of the actuator and therefore is not of control interest.

As a second case, the gain-scheduling controller is designed and employed on the nominal process following the algorithms 1 and 2. Using the method described in the previous section, the minimum disturbance limit is found to be as $w_{\min }=1.1364$ and the disturbance interval is divided into $N=7$ intermediate 
(a)

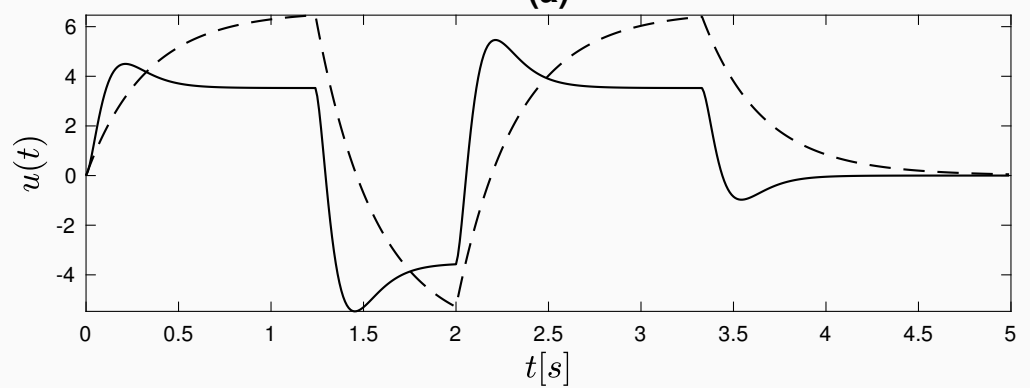

(b)

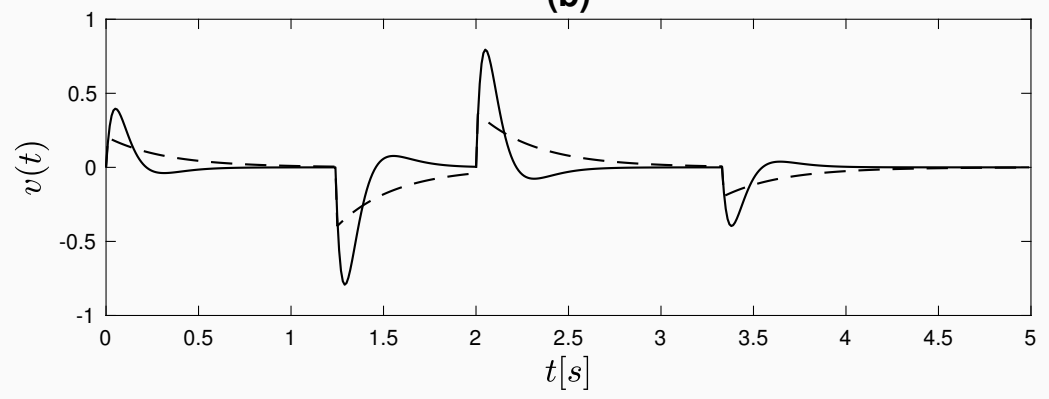

Figure 3: (Nominal case) (a) Variations of the control signals (b) variations of the rate of control signals for the proposed controller (bold) and the LQR (dashed).
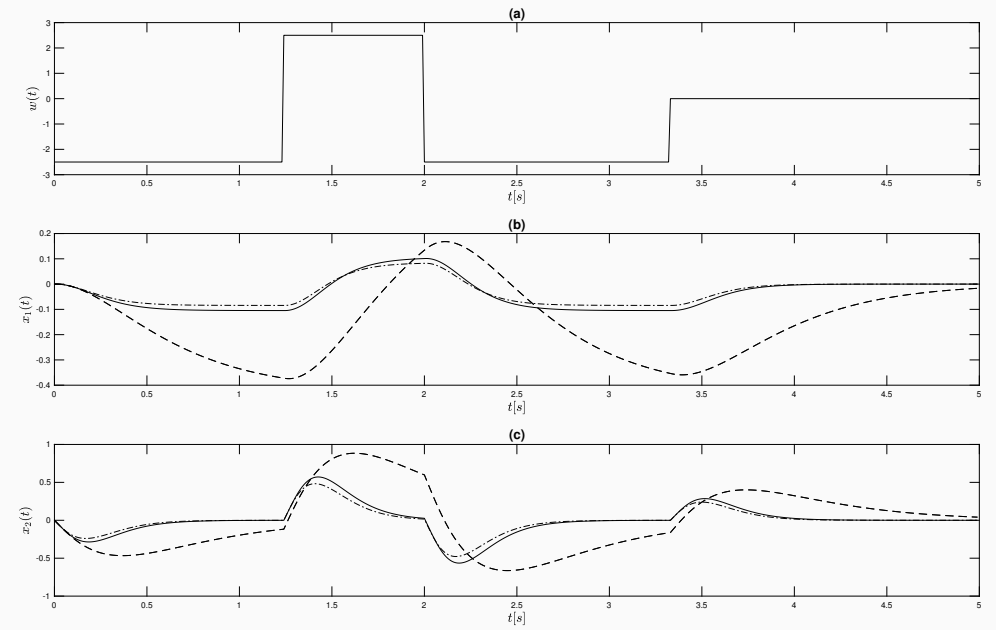

Figure 4: (Nominal case) (a) Variation of the disturbance signal $w(t)$. (b) variation of $x_{1}(t)$ (c) variation of $x_{2}(t)$ for LQR (dashed), proposed controller (solid) and the proposed nominal gain-scheduling controller (dashed-dot). 


\begin{tabular}{lllll}
\hline Disturbance bound & Controller gain $K_{i}$ & \\
\hline$w_{\min }=1.1364$ & $K_{1}=\left[\begin{array}{llll}-98.0740 & -24.0420 & -1.4895 & -0.9994\end{array}\right]$ \\
$w_{2}=1.2500$ & $K_{2}=\left[\begin{array}{llll}-96.0198 & -23.5384 & -1.4608 & -0.9768\end{array}\right]$ \\
$w_{3}=1.3889$ & $K_{3}=\left[\begin{array}{llll}-85.0618 & -20.8705 & -1.3064 & -0.8624\end{array}\right]$ \\
$w_{4}=1.5625$ & $K_{4}=\left[\begin{array}{llll}-72.2972 & -17.7789 & -1.1239 & -0.7380\end{array}\right]$ \\
$w_{5}=1.7857$ & $K_{5}=\left[\begin{array}{llll}-58.0536 & -14.3411 & -0.9186 & -0.6059\end{array}\right]$ \\
$w_{6}=2.0833$ & $K_{6}=\left[\begin{array}{llll}-42.6357 & -10.6328 & -0.6954 & -0.4678\end{array}\right]$ \\
$w_{\infty}=2.5000$ & $K_{7}=\left[\begin{array}{llll}-26.4232 & -6.7361 & -0.4561 & -0.3289\end{array}\right]$ \\
\hline
\end{tabular}

Table 1: (Nominal case) Gain-scheduling control gains for different disturbance levels.

levels. Table 1 shows the intermediate disturbance levels used in the design of the gain scheduling controller and the corresponding controller gains. Applying the gain-scheduling controller on the plant, we obtain slightly better response than the fixed controller developed by considering for the worst case disturbance. Figure 4 shows the response of the system to the gain-scheduling controller together with the fixed controller and the LQR. On the other hand, Figure 5 shows the variations of the control signals together with the controller index history of the gain-scheduling mechanism that is recorded during operation. It is obvious from the variations of the controller signals that a much more aggressive controller is achieved by the implementation of the gain-scheduling mechanism compared to the fixed controller. In the vast majority of the simulation duration, the controller operates at the maximum gain levels unless the time instants $t=$ $1.25 \mathrm{~s}$ and $t=2 \mathrm{~s}$ when the disturbance changes its direction instantaneously and 495 aggressively from one peak to another. To be able to evaluate the performance of the robust controller , the plant is also considered to be uncertain with timevarying parameters $m(k)=1+0.1 \delta_{m}(k), c(k)=0.1+0.02 \delta_{c}(k)$ and $\tau(k)=$ $10+\delta_{\tau}(k)$ where $\delta_{m, c, \tau}(k) \in[-1,1]$. A discrete-time LFT representation is then obtained as in 41) with the uncertainty block $\Delta_{k}=\operatorname{diag}\left(\delta_{c}, \delta_{m}, \delta_{m}, \delta_{\tau}\right)$ and the

following system matrices:

$$
\begin{gathered}
{[\mathcal{A}|\mathcal{B}| \mathcal{H}]=\left[\begin{array}{cccc|c|c}
1 & 0.01 & 0 & 0 & 0 & 0 \\
0.098 & 0.999 & 0.01 & 0 & 0 & 0.01 \\
0 & 0 & 0.9 & 0.1 & 0.1 & 0 \\
0 & 0 & 0 & 1 & 1 & 0
\end{array}\right],} \\
\mathcal{B}_{p}=\left[\begin{array}{cccc}
0 & 0 & 0 & 0 \\
-0.0014 & 0.0008 & 0.0376 & 0 \\
0 & 0 & 0 & 0.1316 \\
0 & 0 & 0 & 0
\end{array}\right]
\end{gathered}
$$



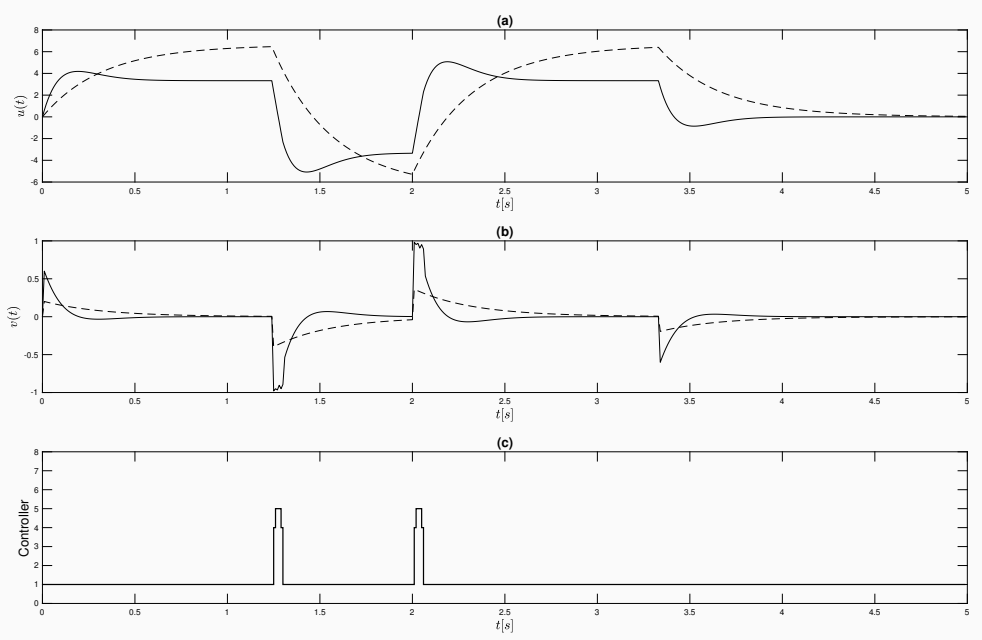

Figure 5: (Nominal case: Gain-scheduling control) (a) Variations of the control signal $u(t),(\mathbf{b})$ rate of the control signal $v(t)$ and (c) the controller index history. (LQR (dashed) and the proposed nominal gain-scheduling controller (solid).

$$
\begin{gathered}
\mathcal{D}_{p}=\left[\begin{array}{cccc}
0 & -0.1189 & 0.0003 & 0 \\
0 & -0.1 & 0 & 0 \\
0 & 0 & -0.1 & 0 \\
0 & 0 & 0 & 0
\end{array}\right], \\
{\left[\mathcal{A}_{d}\left|\mathcal{B}_{d}\right| \mathcal{H}_{d}\right]=\left[\begin{array}{cccc|c|c}
0 & 0.1 & 0 & 0 & 0 & 0 \\
0 & 0.1 & -0.0001 & 0 & 0 & -0.0001 \\
0 & 0 & -0.0266 & 0 & 0 & -0.0266 \\
0 & 0 & -0.076 & 0.076 & 0.076 & 0
\end{array}\right],}
\end{gathered}
$$

and

$$
\boldsymbol{\Delta}=\left\{\Delta^{1}, \ldots, \Delta^{8}\right\}
$$

Due to the structure of the uncertain set $\boldsymbol{\Delta}$, the extreme points of polytopic uncertain set are as follows: $\Delta^{1}=\operatorname{diag}(1,1,1,1), \Delta^{2}=\operatorname{diag}(1,1,1,-1)$, $\Delta^{3}=\operatorname{diag}(1,-1,-1,1), \Delta^{4}=\operatorname{diag}(1,-1,-1,-1), \Delta^{5}=\operatorname{diag}(-1,1,1,1), \Delta^{6}=$ $\operatorname{diag}(-1,1,1,-1), \Delta^{7}=\operatorname{diag}(-1,-1,-1,1)$ and $\Delta^{8}=\operatorname{diag}(-1,-1,-1,-1)$. For the robust controller design, feasible values for the control parameters are found to be as $r_{1}=0.07$ and $r_{2}=0.95$. On the other hand, the optimal controller gain is computed as

$$
K=\left[\begin{array}{llll}
-12.9114 & -3.8350 & -0.2956 & -0.2491
\end{array}\right],
$$

which is highly conservative compared to the controller gains obtained for the nominal case. Figure 6 shows the variations of state trajectories together with 

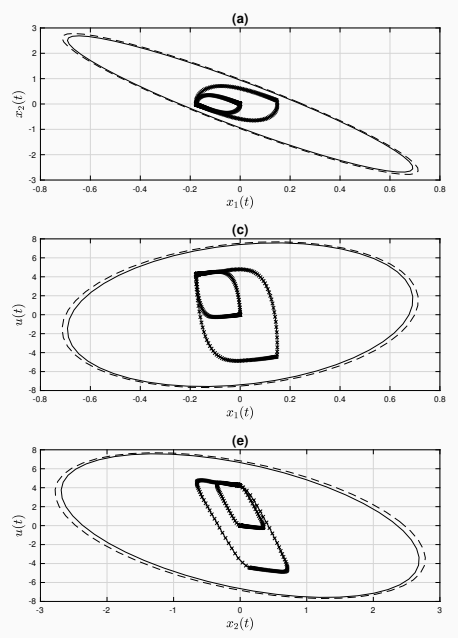
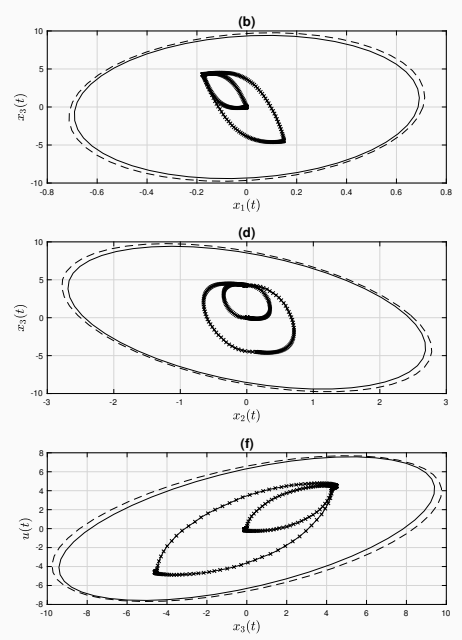

Figure 6: (Uncertain case: robust control) Projection of the closed-loop system trajectories $(-\mathrm{x})$ together with the invariant ellipsoids $\mathcal{E}\left(P^{-1}, w_{\infty}^{2}\right)$ (solid) and $\mathcal{E}\left(Q^{-1}, w_{\infty}^{2}\right)$ (dashed) on the corresponding states.

the bounding ellipsoids. To demonstrate the performance of the proposed robust controller, the variations of the closed-loop state trajectories $x_{1}(t)$ and $x_{2}(t)$ are also provided in Figure 7 along with the LQR. As it can be observed from the figure, there is a small acceptable degradation in the performance of the robust controller when the controlled plant has uncertain time-varying parameters which is expected. At this point, it is worth commenting on performance of the LQR on the uncertain plant. The LQR, which is designed for the nominal system, demonstrates fairly consistent performance to the changes in the system parameters in discrete-time case and minimally affected in terms of its performance. However, the proposed controller still outperforms the LQR in terms of disturbance attenuation and all controller signals stay within the physical limits of the actuator (see Figure 8).

As a final case study, the robust gain-scheduling technique is employed on the uncertain plant. The realisation of the Algorithm 1 yields $r_{1}=0.07$ and $r_{2}=0.95$ for the design parameters. For this example, effective minimum bound for the disturbance signal is found to be as $w_{\min }=0.8333$. Based on this selection, $N=5$ interim controllers are obtained as shown in the Table 2. Figure 7 shows the state trajectories obtained by the robust gain scheduling controller in comparison with the LQR and the robust controller having constant gains. As it can be observed from the figure, application of the gain-scheduling technique slightly improves the performance of the robust controller by elevating the ag${ }_{535}$ gressiveness of the controller. This situation can be observed from the Figure 9 where the variations of the rate of-the-control signal $v(t)$ reaches upper limits, especially during the times when disturbance signal demonstrates big changes. 

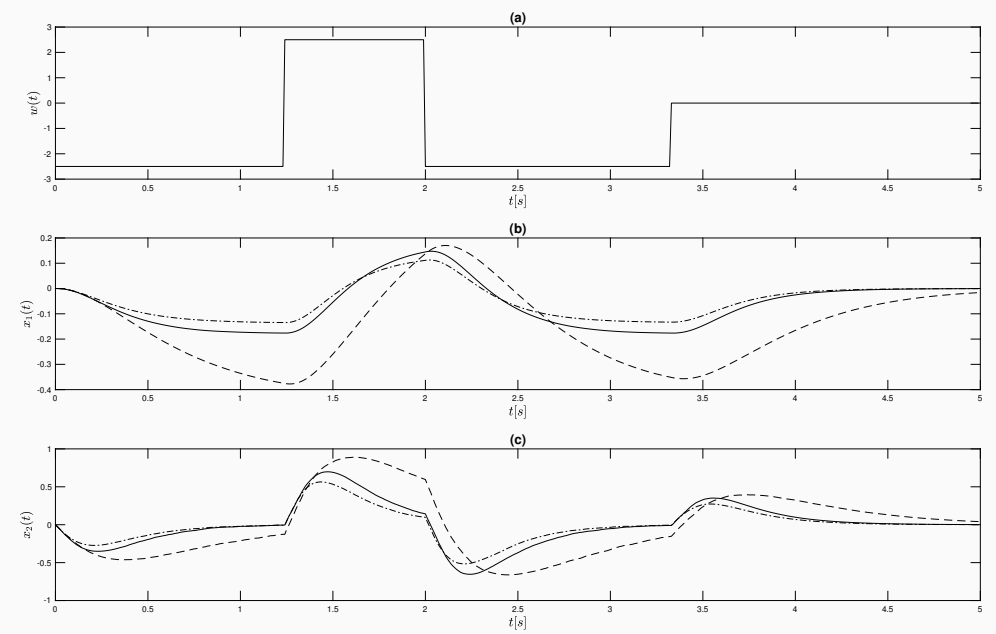

Figure 7: (Uncertain case) (a) Variation of the disturbance signal $w(t)$. (b) variation of $x_{1}(t)$ (c) variation of $x_{2}(t)$ for LQR (dashed), proposed controller (solid) and the proposed robust gain-scheduling controller (dashed-dot).
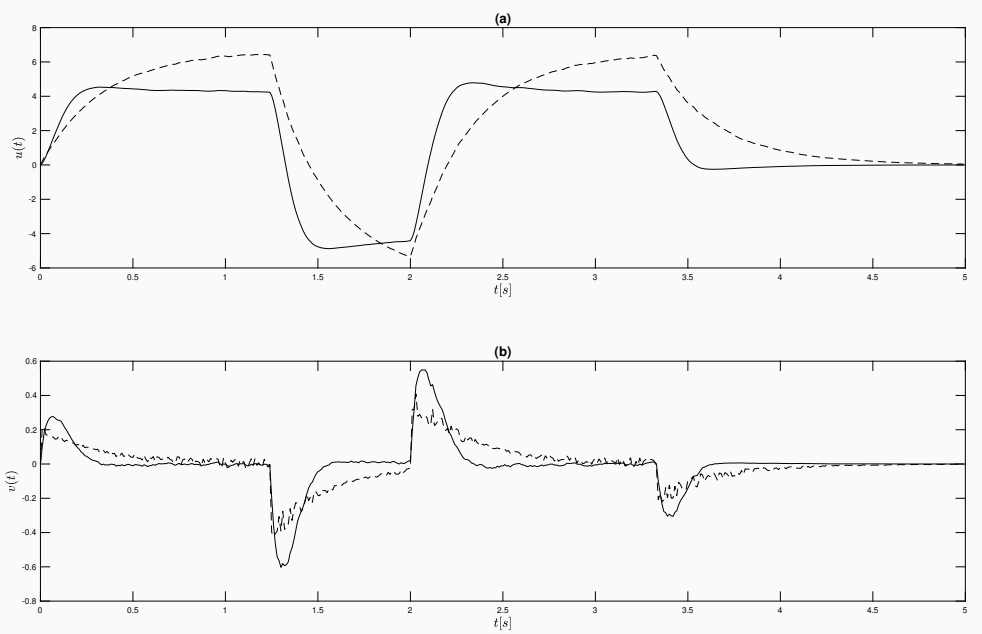

Figure 8: (Uncertain case: Robust control) (a) Variations of the control signals (b) variations of the rate of control signals for the proposed controller (bold) and the LQR (dashed). 

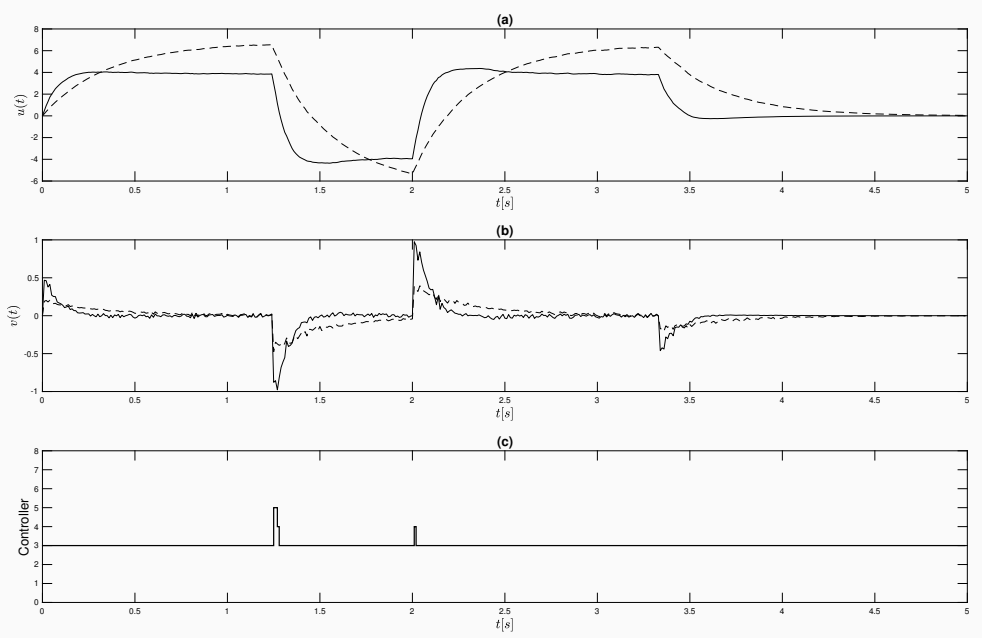

Figure 9: (Uncertain case: Robust gain-scheduling control) (a) Variations of the control signal $u(t),(\mathbf{b})$ rate of the control signal $v(t)$ and (c) the controller index history. (LQR (dashed) and the proposed nominal gain-scheduling controller (solid)).

At those instants, the controller operating with the gain matrix $K_{3}$ switches to lower gains $K_{5}$ and $K_{4}$ respectively to secure $v(t)$ not to saturate. Finally, the contracting ellipsoids $\mathcal{E}\left(Q_{i}^{-1}, w_{\max }^{2}\right), i=1, \ldots, 5$ are shown in Figure 10. It is obvious from the figure that tighter ellipsoids can be obtained for smaller values of $w_{\max }$. However the contracting ellipsoids converge to a limiting ellipsoid in 5 iterations. At this point, it is worth commenting on the limited performance of the gain-scheduling controller for the considered problem. Due to the cruel rate

545 bound $\bar{v}$ of the actuator, the controller has very limited room for operation and the rate of the control signal tends to saturate easily. This situation brings hard limits on the control gains that can be applicable; hence, yielding limited benefit for the control system. This is also obvious from the Figure 10. However, for systems having higher $\bar{v} / \bar{u}$ ratio, the gain scheduling controller would provide considerable contributions to the overall performance.

\section{Conclusion}

We have developed a robust state-feedback controller design method for uncertain discrete-time systems subject to bounded disturbances and having hard magnitude and rate bounds on their inputs. Different from the literature 555 where mostly anti-windup and nested saturation blocks are used, in this note, we considered velocity form of the system associated with two nested invariant and attractive ellipsoids to solve the problem. Here, the inner ellipsoid is used for disturbance rejection purposes whereas the outer ellipsoid is utilized to ensure an unsaturated control for systems having non-zero initial conditions at all 


\begin{tabular}{lllll}
\hline Disturbance bound & Controller gain $K_{i}$ & \\
\hline$w_{\min }=0.83$ & $K_{1}=\left[\begin{array}{llll}-67.7019 & -19.7395 & -1.3988 & -0.9967\end{array}\right]$ \\
$w_{2}=1$ & $K_{2}=\left[\begin{array}{llll}-62.4129 & -18.1989 & -1.3017 & -0.9116\end{array}\right]$ \\
$w_{3}=1.25$ & $K_{3}=\left[\begin{array}{llll}-47.4655 & -13.8521 & -1.0108 & -0.6916\end{array}\right]$ \\
$w_{4}=1.67$ & $K_{4}=\left[\begin{array}{llll}-29.7885 & -8.7253 & -0.6543 & -0.4534\end{array}\right]$ \\
$w_{\infty}=2.5$ & $K_{5}=\left[\begin{array}{llll}-12.9114 & -3.8350 & -0.2956 & -0.2491\end{array}\right]$ \\
\hline
\end{tabular}

Table 2: (Uncertain case: Robust gain-scheduling control) Gain-scheduling control gains for different disturbance levels.
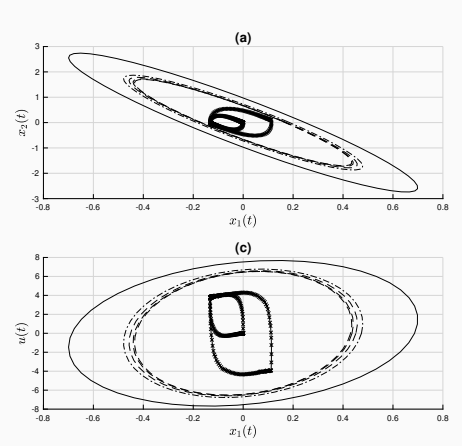

(e)

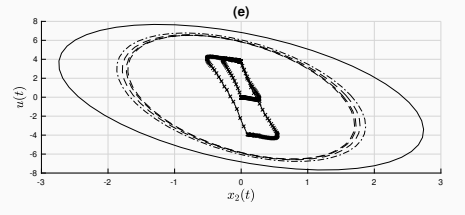

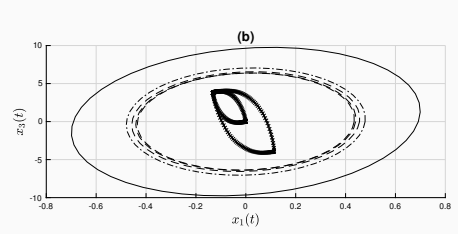
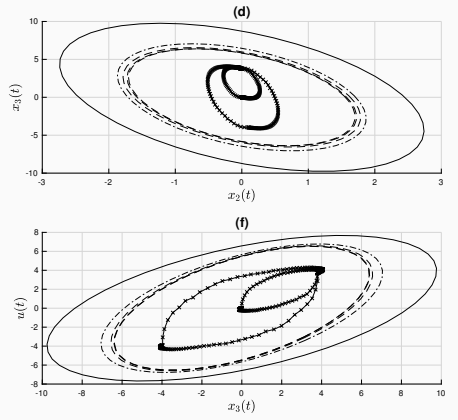

Figure 10: (Uncertain case: Gain-scheduling control) Projections of the closed-loop system trajectories $(-\mathrm{x})$ together with the invariant ellipsoids $\mathcal{E}\left(Q_{i}^{-1}, w_{\infty}^{2}\right) i=1: 5\left(Q_{5}\right.$ (solid), $Q_{4}$ (dash-dot), $Q_{3}, Q_{2}, Q_{1}$ (dash)) on the corresponding states. 
times. Adapting full block S-Procedure, we provided a robust controller design method having minimal conservatism and applicable not only to systems with affine parameter dependence, but any parametric system with rational parameter dependence. Finally, we also provided a modified robust gain-scheduling controller topology which leads to an improvement in the performance by in-

565 creasing the aggressiveness of fixed controller. The paper still lacks of control design methods for systems having performance and measured outputs. This problem is left as a future study as it requires a completely different setting. Also, it would be interesting to extend the method to the solution of nonlinear control problems which can be handled in LPV framework.

\section{References}

[1] P. Chan, S. F. Catpinar, B. Chang, H. Kwatny, C. M. Belcastro, Aircraft spiral dive attractors due to actuator saturation, in: 2017 13th IEEE International Conference on Control Automation (ICCA), 2017, pp. 1072-1077.

[2] Anon, Why the grippen crashed, Aerospace America (1994) 11.

575 [3] G. Stein, Respect the unstable, IEEE Control Systems 23 (4) (2003) 12-25.

[4] M. A. Dornheim, Report pinpoints factors leading to YF-22 crash. (fighter plane prototype), Aviation Week and Space Technology 137 (19) (1992) 53.

[5] Y. Wang, R. M. Murray, Bifurcation control of rotating stall with actuator magnitude and rate limits: Part imodel reduction and qualitative dynamics, Automatica 38 (4) (2002) $597-610$.

[6] P.-C. Chen, J. S. Shamma, Gain-scheduled $\ell$ 1-optimal control for boilerturbine dynamics with actuator saturation, Journal of Process Control 14 (3) (2004) $263-277$.

[7] I. B. Kucukdemiral, F. Cakici, H. Yazici, A model predictive vertical motion control of a passenger ship, Ocean Engineering 186 (2019) 106100.

[8] A. Stoorvogel, A. Saberi, Editorial (special issue on control problems with constraints), International Journal of Robust and Nonlinear Control 9 (10) (1999) 583-584.

[9] J. Yang, T. Li, C. Liu, S. Li, W. Chen, Nonlinearity estimator-based control of a class of uncertain nonlinear systems, IEEE Transactions on Automatic Control (2019) 1-1.

[10] S. Galeani, S. Tarbouriech, M. Turner, L. Zaccarian, A tutorial on modern anti-windup design, European Journal of Control 15 (3-4) (2009) 418-440.

[11] T. Hu, Z. Lin, Control Systems with Actuator Saturation: Analysis and Design, Birkhauser Boston, Inc., Secaucus, NJ, USA, 2001. 
[12] V. Kapila, K. Grigoriadis (Eds.), Actuator saturation control, Marcel Dekker, 2002.

[13] P. Lu, Tracking control of nonlinear systems with bounded controls and control rates, Automatica 33 (6) (1997) 1199 - 1202.

[14] P.-C. Chen, Multi-objective control of nonlinear boiler-turbine dynamics with actuator magnitude and rate constraints, ISA Transactions 52 (1) (2013) 115-128.

[15] V. Azhmyakov, M. Mera, R. Jurez, Advances in attractive ellipsoid method for robust control design, International Journal of Robust and Nonlinear Control 29 (5) (2019) 1418-1436.

[16] K. Kefferptz, B. Fischer, J. Adamy, A nonlinear controller for input amplitude and rate constrained linear systems, IEEE Transactions on Automatic Control 58 (10) (2013) 2693-2697.

[17] D. Q. Mayne, Model predictive control: Recent developments and future promise, Automatica 50 (12) (2014) 2967-2986.

[18] J. Yang, H. Wu, L. Hu, S. Li, Robust predictive speed regulation of converter-driven dc motors via a discrete-time reduced-order gpio, IEEE Transactions on Industrial Electronics 66 (10) (2019) 7893-7903.

[19] F. A. Bender, J. M. Gomes da Silva Jr., Output feedback controller design for systems with amplitude and rate control constraints, Asian Journal of Control 14 (4) (2012) 1113-1117.

[20] S. Galeani, S. Onori, A. Teel, L. Zaccarian, A magnitude and rate saturation model and its use in the solution of a static anti-windup problem, Systems \& Control Letters 57 (1) (2008) 1-9.

${ }_{620}$ [21] M. S. Reineh, S. S. Kia, F. Jabbari, New anti-windup structure for magnitude and rate limited inputs and peak-bounded disturbances, Automatica 97 (2018) 301-305.

[22] A. Bateman, Z. Lin, An analysis and design method for linear systems under nested saturation, Systems and Control Letters 48 (1) (2003) 41-52.

625 [23] A. H. K. Palmeira, J. M. Gomes Da Silva, S. Tarbouriech, I. M. F. Ghiggi, Sampled data control under magnitude and rate saturating actuators, International Journal of Robust and Nonlinear Control 26 (15) (2016) 32323252 .

[24] S. Tarbouriech, C. Prieur, J. Da Silva, Stability analysis and stabilization of systems presenting nested saturations, IEEE Transactions on Automatic Control 51 (8) (2006) 1364-1371.

[25] B. Zhou, Analysis and design of discrete-time linear systems with nested actuator saturations, Systems \& Control Letters 62 (10) (2013) 871 - 879. 
[26] I. E. Köse, F. Jabbari, Rate and magnitude-bounded actuators: Scheduled state feedback design, IFAC Proceedings Volumes 35 (1) (2002) 73 - 78, 15th IFAC World Congress.

[27] F. Jabbari, I. E. Köse, Rate and magnitude-bounded actuators: scheduled output feedback design, International Journal of Robust and Nonlinear Control 14 (13-14) (2004) 1169-1184.

[28] V. S. Chellaboina, W. M. Haddad, J. H. Oh, Fixed-order dynamic compensation for linear systems with actuator amplitude and rate saturation constraints, International Journal of Control 73 (12) (2000) 1087-1103.

[29] A. Poznyak, A. Polyakov, V. Azhmyakov, Attractive Ellipsoids in Robust Control, Birkhäuser, Basel, 2014.

[30] L. Wang, A tutorial on model predictive control: Using a linear velocityform model, Developments in Chemical Engineering and Mineral Processing 12 (56) (2004) 573-614.

[31] C. W. Scherer, A full block s-procedure with applications, in: Proceedings of the 36th IEEE Conference on Decision and Control, Vol. 3, 1997, pp. $2602-2607$.

[32] P. Garcia, K. Ampountolas, Robust disturbance rejection by the attractive ellipsoid method part II: Discrete-time systems, IFAC Papers On Line 51 (32) (2018) 93-98.

[33] S. Boyd, L. El Ghaoui, E. Feron, V. Balakrishnan, Linear Matrix Inequalities in System and Control Theory, Vol. 15 of Studies in Applied Mathematics, SIAM, Philadelphia, PA, 1994.

[34] A. Zemouche, R. Rajamani, B. Boulkroune, H. Rafaralahy, M. Zasadzinski, $\mathcal{H}_{\infty}$ circle criterion observer design for Lipschitz nonlinear systems with enhanced LMI conditions, in: Proceedings of the 2016 American Control Conference (ACC), 2016, pp. 131-136.

[35] R. A. Horn, C. R. Johnson, Matrix Analysis, 2nd Edition, Cambridge University Press, New York, NY, USA, 2012.

[36] J. Doyle, A. Packard, K. Zhou, Review of LFTs, LMIs, and $\mu$, in: Proceedings of the 30th IEEE Conference on Decision and Control, IEEE, 1991, pp. $1227-1232$.

[37] C. Scherer, S. Weiland, Lecture notes: Linear matrix inequalities in control, Dutch Institute for Systems and Control, Delft, The Netherlands, 2015.

[38] A. Gupta, H. Koroglu, P. Falcone, Computation of low-complexity controlinvariant sets for systems with uncertain parameter dependence, Automatica 101 (2019) $330-337$. 
[39] P. A. Parrilo, Structured Semidefinite Programs and Semialgebraic Geometry Methods in Robustness and Optimization, $\mathrm{PhD}$ thesis, California Institute of Technology, 2000.

[40] C. Scherer, LMI relaxations in robust control, European Journal of Control 12 (1) (2006) $3-29$.

[41] H. Koroglu, C. Scherer, Robust stability analysis against perturbations of smoothly time-varying parameters, in: Proceedings of the 45th IEEE Conference on Decision and Control, IEEE, 2006, pp. 2895-2900.

[42] S. Sajjadi-Kia, F. Jabbari, Use of scheduling for anti-windup controller design, in: 2007 American Control Conference, IEEE, 2007-07, pp. 51945199 .

[43] J. Löfberg, YALMIP : A toolbox for modeling and optimization in MATLAB, in: In Proceedings of the CACSD Conference, Taipei, Taiwan, 2004. 